\title{
Effect of Crop Geometry and Foliar Nutrition on Growth and Yield of Irrigated Blackgram (Vigna mungo L.)
}

\author{
S. Rajeshkumar ${ }^{1}$, S. Nalliah Durairaj ${ }^{1}$ and V. Kannan ${ }^{2 *}$ \\ ${ }^{1}$ Department of Agronomy, Agricultural College and Research Institute, \\ Killikulam, Tamil Nadu (628 252), India \\ ${ }^{2}$ Department of Agronomy, Agricultural College and Research Institute, \\ Madurai, Tamil Nadu (625 104), India \\ *Corresponding author
}

\begin{abstract}
A B S T R A C T
A field experiment was carried out during Rabi season of 2012- 13, at Agricultural College and Research Institute, Killikulam, to evaluate the suitability and economic viability of crop geometry and foliar nutrition on growth and yield of irrigated blackgram.

Keywords

Foliar nutrition, Crop geometry, TNAU Pulse wonder, Black gram.

Article Info

Accepted:

28 September 2017

Available Online:

10 November 2017

The experiment was laid out in Randomized block design with three replications, comprising fifteen treatments. The variety used was VBN (Bg) 5. Results revealed that Crop geometry at $40 \times 30 \mathrm{~cm}$ with foliar spray of TNAU Pulse Wonder at $1.125 \%$ recorded for better growth characters of individual plant viz., plant height, number of branches plant $^{-1}$, number of root nodules plant ${ }^{-1}$, Light interception and Yield attributes of individual plant like number of pod clusters plant ${ }^{-1}$, number of pods cluster ${ }^{-1}$, pod length and number of seeds pod $^{-1}$. In the case of dry matter production $\mathrm{kg} \mathrm{ha}^{-1}$ and leaf area index of growth attributing characters, grain, bhusa and haulm yield $\mathrm{kg} \mathrm{ha}^{-1}$ were recorded higher with crop geometry of $30 \times 10 \mathrm{~cm}$ with foliar spray of TNAU Pulse Wonder at $1.125 \%$. The yield levels were on par with crop geometry of $30 \times 20 \mathrm{~cm}$ with same application. The uptake of nutrients was also higher under crop geometry of $30 \times 10 \mathrm{~cm}$ as well as $30 \times 20$ $\mathrm{cm}$ with foliar spray of TNAU Pulse Wonder at $1.125 \%$. The highest net return and B: C ratio were obtained when the crop geometry at $30 \times 20 \mathrm{~cm}$ with foliar spray of TNAU Pulse Wonder at $1.125 \%$.
\end{abstract}

\section{Introduction}

Pulses are the major source of dietary protein in the vegetarian diet of our country. They occupy a unique position in Indian agriculture by virtue of the fact that they constitute a major and the only high protein component to the average Indian diet. They also supply nutritive fodder and maintain soil fertility through biological nitrogen fixation and thus, play a vital role in furthering sustainable agriculture. Popularity of Blackgram (Vigna mungo L.) is mainly because of its superior nutritional quality and can very well fit into multiple cropping systems, because of short duration. It can be grown as intercrop with pigeonpea, maize, sorghum, cotton and sugarcane. It can also be grown as a green manure and fodder crop.

In India, the total area under black gram is $3.06 \mathrm{M}$. ha with a production of $1.70 \mathrm{Mt}$ and a 
productivity $555 \mathrm{~kg} \mathrm{ha}^{-1}$ during 2014 (Anon, 2014). In Tamil Nadu, the total area under black gram is 94.8 ('000 ha) with a production 160.4 ('000 tones) and productivity $406 \mathrm{~kg} \mathrm{ha}^{-1}$ during 2015 (Anon, 2015).

The System of Rice Intensification (SRI) is a novel method originated in Madagascar during 1983 and spread all over the world. The SRI has set of principles that was used in rice and many success stories beyond the technologies. In the recent past, the success of SRI principles are being extrapolated to other crops such as wheat, maize, sorghum, finger millet, soybean, greengram, blackgram, kidney bean, lentil, mustard, sugarcane, tomato, brinjal, chilli, potato, carrot and onion in the name of System of Crop Intensification (SCI) (ISD, 2009). Similar to SRI, the SCI practices also proved to increase the yield level of crops more than two times (Uphoff et al., 2011). In pursuit of extending the beneficial effect of SRI to SCI in blackgram, the present study was programmed with different crop geometry.

And also in agriculture practices, fertilizer is an important source to increase crop yields. Among fertilizer application methods, one of the most important methods of application is foliar nutrition, because foliar nutrients facilitate easy and quick absorption of nutrients by penetrating the stomata or leaf cuticle and enters the cells. Due to several compensations of foliar application methods like quick and proficient response to needs of plants, less needed products and soil conditions independency, the concentration towards foliar fertilizers is arising day by day. It is also determined that during crop growth supplementary foliar fertilization increase plant mineral status and improve crop yields. With this background, an investigation was carried out to test the crop geometry and foliar nutrition in blackgram.

\section{Materials and Methods}

The field experiment was carried out during Rabi season of November 2012 - January 2013, at Agricultural College and Research Institute, Killikulam. The experimental field is geographically located in the southern part of Tamil Nadu at $8^{\circ} 46^{\prime} \mathrm{N}$ latitude and $77^{\circ} 42^{\prime}$ E longitude at an altitude of 40 meters above mean sea level. The climate of the experimental site is semi-arid tropical type. The mean annual rainfall is $786.6 \mathrm{~mm}$ in 40 rainy days. The mean maximum and minimum temperature of the location are $33.4^{\circ} \mathrm{C}$ and $23.6^{\circ} \mathrm{C}$ respectively. The relative humidity ranges from 60 to 80 per cent. The texture of the surface soil $(0-15 \mathrm{~cm})$ of the experimental field was sandy clay loam. The fertility status was neutral (7.1) in $\mathrm{pH}$ (Jackson, 1973), high in organic carbon (0.59 $\%$ ) (Walkley and Black, 1934) low in available nitrogen $\left(270 \mathrm{~kg} \mathrm{~N}^{-1}\right.$ ) (Subbiah and Asija, 1956), medium in available phosphorus (13 kg $\mathrm{P}_{2} \mathrm{O}_{5} \mathrm{ha}^{-1}$ ) (Olsen et al., 1954) and potassium (233 $\mathrm{kg} \mathrm{K} \mathrm{K}_{2} \mathrm{O} \mathrm{ha}^{-1}$ ) (Stanford and English, 1949). The climate of the experimental site is semi-arid tropical type and annual rainfall is $786.6 \mathrm{~mm}$ in 40 rainy days. The experiment was laid out in Randomized block design with three replications, comprising fifteen treatments viz., $\mathrm{T}_{1}-30 \times 10 \mathrm{~cm}, \mathrm{~T}_{2}-30 \times 20 \mathrm{~cm}, \mathrm{~T}_{3}-30 \times$ $30 \mathrm{~cm}, \mathrm{~T}_{4^{-}} 40 \times 20 \mathrm{~cm}, \mathrm{~T}_{5^{-}} 40 \times 30 \mathrm{~cm}, \mathrm{~T}_{6^{-}}$ $30 \times 10 \mathrm{~cm}+$ fs TNAU Pulse Wonder at $1.125 \%, \mathrm{~T}_{7}-30 \times 20 \mathrm{~cm}+$ fs TNAU Pulse Wonder at $1.125 \%, \mathrm{~T}_{8}-30 \times 30 \mathrm{~cm}+\mathrm{fs}$ TNAU Pulse Wonder at $1.125 \%, \mathrm{~T}_{9^{-}} 40 \times 20$ $\mathrm{cm}+\mathrm{fs}$ TNAU Pulse Wonder at $1.125 \%, \mathrm{~T}_{10^{-}}$ $40 \times 30 \mathrm{~cm}+$ fs TNAU Pulse Wonder at $1.125 \%, \mathrm{~T}_{11}-30 \times 10 \mathrm{~cm}+$ fs DAP at $2 \%$, $\mathrm{T}_{12^{-}} 30 \times 20 \mathrm{~cm}+$ fs DAP at $2 \%, \mathrm{~T}_{13^{-}} 30 \times 30$ $\mathrm{cm}+$ fs DAP at $2 \%, \mathrm{~T}_{14^{-}} 40 \times 20 \mathrm{~cm}+\mathrm{fs}$ DAP at $2 \%, \mathrm{~T}_{15^{-}} 40 \times 30 \mathrm{~cm}+$ fs DAP at $2 \%$. The foliar spray was given twice at flowering stage and 15 days later. $\mathrm{VBN}(\mathrm{Bg}) 5$ variety was used as test variety. The recommended 
dose of 25: $50: 25: 25 \mathrm{~kg}$ of NPK and $\mathrm{ZnSO}_{4}$ $\mathrm{ha}^{-1}$ was applied in the form of urea $(46 \% \mathrm{~N})$, single super phosphate $\left(\begin{array}{lll}16 \% & \mathrm{P}_{2} \mathrm{O}_{5}\end{array}\right)$ and muriate of potash $\left(60 \% \mathrm{~K}_{2} \mathrm{O}\right)$. The fertilizers were applied uniformly to all plots at the time of sowing. Foliar spray of DAP or TNAU Pulse Wonder was given on 30 and 45 DAS as per the treatment schedule.

The observations were recorded on growth and growth analysis parameters such as number of branches plant ${ }^{-1}$, number of root nodules plant ${ }^{-1}$, Dry matter production $\mathrm{kg}$ ha ${ }^{1}$ and growth analysis parameters LAI, CGR Light interception. The yield parameters of pod clusters plant ${ }^{-1}$, number of pods cluster ${ }^{-1}$, pod length and number of seeds pod 1 , grain, bhusa and haulm yield $\mathrm{kg} \mathrm{ha}^{-1}$. The observed data on crop were statistically analyzed based on the procedure given by Gomez and Gomez (2010). Critical differences were worked out at five per cent probability level wherever the treatments were significant. The treatment differences that were non-significant were denoted as NS.

\section{Results and Discussion}

\section{Influence of crop geometry and foliar nutrition on growth characters}

The plant growth is manifested in many ways. Though growth characters viz., plant height, number of branches plant $^{-1}$, root nodules plant $^{-1}$ and dry matter production are largely genetically controlled, can also be altered agronomically by manipulating environment. The plant height is a direct index to measure the growth and vigour of plants. In general, the plant height gradually increased from initial stage to harvest stage. The plant height was not physically affected due to crop geometry and foliar nutrition at 20 DAS. However, an increasing trend with wider crop geometry could be noticed during all growth stages.
The plant height is a direct index to measure the growth and vigour of plants. In general, the plant height gradually increased from initial stage to harvest stage. The plant was not physically affected due to crop geometry. However, an increasing trend with closer geometry level could be noticed during all growth stages (Table. 2). This may be due to the competition between the inter and intra plants for sun light, water, nutrients and space at closer spacing which encouraged selfthinning of branches and enhanced vertical growth rather than horizontal growth in Crop geometry of $40 \times 30$. Similar findings were also reported by Kumar and Badiyala (2005) in soybean and Siddharaju et al., (2010) in cluster bean. Crop geometry of $40 \times 30 \mathrm{~cm}$ produced significantly more number of branches plant ${ }^{-1}$ at 50 DAS. This might be due to more space for nutrient absorption in presence of proper soil moisture content during growth period. Similar results have also been reported by Sarkar et al., (2004). Crop geometry of $40 \times 30 \mathrm{~cm}$ significantly increased the number of root nodules plant $^{-1}$ at 50 DAS. The root nodules increased with decrease in plant densities which might be due to greater root proliferation. Thus increased spacing significantly influenced the growth and root nodulation. These findings are in conformity with Ihsanullah et al., (2002) in mungbean.

The dry matter production of a crop reflects its efficiency in utilizing the available resources. Dry matter production increased steadily with advancing growth stages and reached the maximum at harvest. The DMP $\left(\mathrm{kg} \mathrm{ha}{ }^{-1}\right)$ was found to be more with closer spacing of $30 \times 10 \mathrm{~cm}$, which could be attributed to higher population and accumulation of nutrients unit $\operatorname{area}^{-1}$ compared to wider spacing. This is in accordance with earlier findings of Vijayakumar et al., (2006) in rice and Sathyamoorthi et al., (2008) in greengram 
Influence of crop geometry and foliar nutrition on physiological parameters in blackgram

Agronomic manipulation alters crop physiology to certain extent to derive higher economic product. Physiological attributes like LAI, CGR and LI differed with crop geometry and foliar nutrition in blackgram. Plant stature with broader leaves was observed when blackgram was raised at wider spacings than closer spacings. Though broader leaves and better plant stature have direct relationship with LAI, it could not compensate the cumulative leaf area obtained with more number of plants unit area $^{-1}$ in closer spacing. Crop geometry at $30 \times 10 \mathrm{~cm}$ produced maximum LAI. This might be attributed to higher number of leaves plant $^{-1}$, which resulted in more leaves leading to higher LAI. (Table.3.)This is in conformity with the findings of Sathyamoorthi et al., (2008) in greengram and Cheghakhor et al., (2009) in chickpea.

Crop growth rate makes the assessment of crop productivity unit land ${ }^{-1}$. The CGR was significantly higher with closer crop geometry of $30 \times 10 \mathrm{~cm}$ all the growth stages, which was mainly due to more population unit area 1. Similar findings were also reported by Kathirvelan and Kalaiselvan (2006) and Sathyamoorthi et al., (2008) in greengram.

Light interception was higher at vegetative and flowering stage crop geometry of $40 \times 30$ $\mathrm{cm}$. Higher plant density significantly had greatest dry matter productivity and fraction intercepted photosynthetically active radiation and lowest extinction light coefficient in reproductive stage. Similar findings were also reported by Cheghakhor et al., (2009).

Growth analytical characters such as LAI and CGR were higher with foliar spray of $1.125 \%$ TNAU Pulse Wonder. The higher leaf area index might be due to positive influence of foliar spray of nutrients on cell division and cell elongation which facilitates better crop growth and development resulting in higher growth characters. These results are in line with Santosh Kumar Meena (2004).

\section{Influence of crop geometry and foliar nutrition on yield attributes in blackgram}

Crop geometry at various levels of spacing significantly influenced the yield attributes like number of pod clusters plant ${ }^{-1}$, number of pods cluster $^{-1}$, pod length, number of seeds $\operatorname{pod}^{-1}$ and test grain weight (Table.4.). Number of pod clusters plant ${ }^{-1}$ was significantly higher with wider crop geometry of $40 \times 30 \mathrm{~cm}$ when compared to closer crop geometry. Decreased number of pod clusters plant $^{-1}$ under closer spacing was due to mortality caused by mutual shading during pre-flowering stage of the crop. Similar findings were also reported by Siddaraju et al., (2010) in cluster bean.

Crop geometry at $40 \times 30 \mathrm{~cm}$ recorded the highest number of pods cluster ${ }^{-1}$ and number of seeds $\operatorname{pod}^{-1}$ as compared to closer spacing. The increase in number of pods cluster ${ }^{-1}$ and seeds pod $^{-1}$ at wider crop geometry was due to better crop growth, more space available for plants, lesser competition for moisture and nutrients between plants. This is in conformity with the findings of Bhairappanavar et al., (2005) in blackgram, Nadeem et al., (2004) in mungbean, Goyal et al., (2010) and Siddaraju et al., (2010) in cluster bean.

Planting geometry significantly affected the length of green pods. Pod length was also higher at crop geometry of $40 \times 30 \mathrm{~cm}$. These results are in accordance with Muhammad Amjad et al., (2001), who reported that the longest pods were recorded with widest spacing. 
Table.1 The foliar spray solution details are given below

\begin{tabular}{lll}
\hline Name of the chemical & Concentration & Quantity \\
\hline DAP & $2 \%$ & $20 \mathrm{~g} / \mathrm{lit}$. \\
TNAU Pulse Wonder & $1.125 \%$ & $11.25 \mathrm{~g} / \mathrm{lit}$. \\
\hline
\end{tabular}

Table.2 Influence of crop geometry and foliar nutrition on growth characters in black gram

\begin{tabular}{|c|c|c|c|c|c|c|c|c|c|c|}
\hline \multirow[b]{2}{*}{ Treatments } & \multicolumn{4}{|c|}{ Plant Height } & \multirow{2}{*}{$\begin{array}{c}\text { No. of } \\
\text { branches } \\
\text { plant }^{-1}\end{array}$} & \multirow{2}{*}{$\begin{array}{c}\text { No. of root } \\
\text { nodules } \\
\text { plant }^{-1}\end{array}$} & \multicolumn{4}{|c|}{ DMP $\left(\mathrm{kg} \mathrm{ha}^{-1}\right)$} \\
\hline & $\begin{array}{c}20 \\
\text { DAS }\end{array}$ & $\begin{array}{c}35 \\
\text { DAS }\end{array}$ & $\begin{array}{c}50 \\
\text { DAS }\end{array}$ & Harvest & & & $\begin{array}{l}20 \\
\text { DAS }\end{array}$ & $\begin{array}{l}35 \\
\text { DAS }\end{array}$ & $\begin{array}{l}\text { 50 } \\
\text { DAS }\end{array}$ & Harvest \\
\hline $\mathrm{T}_{1}-30 \times 10 \mathrm{~cm}$ & 15.5 & 29.6 & 38.4 & 48.6 & 3.2 & 20.0 & 68 & 854 & 3778 & 4299 \\
\hline $\mathrm{T}_{2}-30 \times 20 \mathrm{~cm}$ & 15.5 & 30.8 & 39.8 & 49.3 & 3.5 & 22.7 & 62 & 843 & 3521 & 4034 \\
\hline $\mathrm{T}_{3}-30 \times 30 \mathrm{~cm}$ & 15.6 & 31.9 & 40.5 & 49.8 & 3.5 & 23.2 & 40 & 617 & 2483 & 2935 \\
\hline $\mathrm{T}_{4}-40 \times 20 \mathrm{~cm}$ & 15.7 & 32.4 & 40.1 & 50.9 & 3.7 & 24.6 & 53 & 732 & 2912 & 3377 \\
\hline $\mathrm{T}_{5}-40 \times 30 \mathrm{~cm}$ & 15.8 & 33.5 & 42.8 & 51.7 & 3.9 & 25.4 & 48 & 599 & 2646 & 3096 \\
\hline $\mathrm{T}_{6}-30 \times 10 \mathrm{~cm}+1.125 \%$ Pulse Wonder & 15.4 & 33.5 & 45.2 & 57.2 & 5.1 & 25.2 & 88 & 985 & 4528 & 5163 \\
\hline $\mathrm{T}_{7}-30 \times 20 \mathrm{~cm}+1.125 \%$ Pulse Wonder & 15.5 & 35.2 & 45.3 & 58.5 & 5.6 & 26.7 & 86 & 965 & 4361 & 4978 \\
\hline $\mathrm{T}_{8}-30 \times 30 \mathrm{~cm}+1.125 \%$ Pulse Wonder & 15.6 & 36.7 & 46.3 & 58.7 & 5.4 & 27.0 & 65 & 843 & 3550 & 4108 \\
\hline $\mathrm{T}_{9}-40 \times 20 \mathrm{~cm}+1.125 \%$ Pulse Wonder & 15.7 & 36.8 & 47.8 & 59.5 & 5.6 & 27.3 & 79 & 912 & 4143 & 4709 \\
\hline $\mathrm{T}_{10}-40 \times 30 \mathrm{~cm}+1.125 \%$ Pulse Wonder & 15.7 & 37.5 & 48.3 & 61.2 & 5.9 & 28.0 & 68 & 852 & 3555 & 4020 \\
\hline $\mathrm{T}_{11}-30 \times 10 \mathrm{~cm}+2 \% \mathrm{DAP}$ & 15.2 & 32.3 & 43.2 & 54.5 & 4.4 & 23.4 & 70 & 960 & 4150 & 4773 \\
\hline $\mathrm{T}_{12}-30 \times 20 \mathrm{~cm}+2 \% \mathrm{DAP}$ & 15.4 & 33.6 & 43.6 & 55.2 & 4.9 & 24.2 & 66 & 940 & 4089 & 4712 \\
\hline $\mathrm{T}_{13}-30 \times 30 \mathrm{~cm}+2 \% \mathrm{DAP}$ & 15.5 & 34.2 & 44.7 & 56.4 & 4.7 & 25.8 & 49 & 680 & 3264 & 3756 \\
\hline $\mathrm{T}_{14}-40 \times 20 \mathrm{~cm}+2 \% \mathrm{DAP}$ & 15.6 & 34.8 & 44.3 & 55.8 & 4.9 & 26.1 & 65 & 812 & 3591 & 4060 \\
\hline $\mathrm{T}_{15}-40 \times 30 \mathrm{~cm}+2 \% \mathrm{DAP}$ & 15.8 & 35.4 & 45.9 & 57.3 & 5.2 & 26.6 & 65 & 799 & 3339 & 3790 \\
\hline If & 0.5 & 1.0 & 1.4 & 1.7 & 0.14 & 0.78 & 2 & 26 & 116 & 133 \\
\hline $\mathrm{CD}(\mathrm{P}=0.05)$ & NS & 2.1 & 2.8 & 3.5 & 0.30 & 1.62 & 4 & 54 & 238 & 273 \\
\hline
\end{tabular}

Table.3 Influence of crop geometry and foliar nutrition on Physiological parameters of black gram

\begin{tabular}{|c|c|c|c|c|c|c|c|c|}
\hline \multirow[b]{2}{*}{ Treatments } & \multicolumn{2}{|c|}{ LAI } & \multicolumn{3}{|c|}{ Crop growth rate $\left(\mathrm{kg} \mathrm{ha}^{-1}\right.$ day $\left.^{-1}\right)$} & \multicolumn{3}{|c|}{ Light Interception } \\
\hline & $\begin{array}{c}35 \\
\text { DAS } \\
\end{array}$ & $\begin{array}{c}50 \\
\text { DAS }\end{array}$ & $\begin{array}{c}20-35 \\
\text { DAS }\end{array}$ & $\begin{array}{c}\text { 35- 50 } \\
\text { DAS }\end{array}$ & $\begin{array}{l}\text { 50 DAS- } \\
\text { Harvest }\end{array}$ & $\begin{array}{l}\text { Vegetati } \\
\text { ve }\end{array}$ & \begin{tabular}{|l|} 
Flowerin \\
g \\
\end{tabular} & $\begin{array}{l}\text { Maturi } \\
\text { ty }\end{array}$ \\
\hline $\mathrm{T}_{1}-30 \times 10 \mathrm{~cm}$ & 4.24 & 4.97 & 52.4 & 194.9 & 34.7 & 55 & 41 & 21 \\
\hline $\mathrm{T}_{2}-30 \times 20 \mathrm{~cm}$ & 3.70 & 4.68 & 52.1 & 178.5 & 34.2 & 61 & 47 & 23 \\
\hline $\mathrm{T}_{3}-30 \times 30 \mathrm{~cm}$ & 1.87 & 2.44 & 38.5 & 124.4 & 30.1 & 65 & 53 & 25 \\
\hline $\mathrm{T}_{4}-40 \times 20 \mathrm{~cm}$ & 2.07 & 2.05 & 45.3 & 145.3 & 31.0 & 71 & 56 & 29 \\
\hline $\mathrm{T}_{5}-40 \times 30 \mathrm{~cm}$ & 1.49 & 1.71 & 36.7 & 136.5 & 30.0 & 75 & 60 & 31 \\
\hline $\mathrm{T}_{6}-30 \times 10 \mathrm{~cm}+1.125 \%$ Pulse Wonder & 4.64 & 5.54 & 59.8 & 236.2 & 42.3 & 63 & 49 & 27 \\
\hline $\mathrm{T}_{7}-30 \times 20 \mathrm{~cm}+1.125 \%$ Pulse Wonder & 3.97 & 5.39 & 58.6 & 226.4 & 41.1 & 69 & 53 & 29 \\
\hline $\mathrm{T}_{8}-30 \times 30 \mathrm{~cm}+1.125 \%$ Pulse Wonder & 2.01 & 3.15 & 51.9 & 180.5 & 37.2 & 73 & 61 & 31 \\
\hline $\mathrm{T}_{9}-40 \times 20 \mathrm{~cm}+1.125 \%$ Pulse Wonder & 2.16 & 2.72 & 55.5 & 215.4 & 37.7 & 79 & 64 & 37 \\
\hline $\mathrm{T}_{10}-40 \times 30 \mathrm{~cm}+1.125 \%$ Pulse Wonder & 1.65 & 2.03 & 52.3 & 180.2 & 31.0 & 83 & 69 & 39 \\
\hline $\mathrm{T}_{11}-30 \times 10 \mathrm{~cm}+2 \% \mathrm{DAP}$ & 4.49 & 5.24 & 59.3 & 212.7 & 41.5 & 58 & 45 & 24 \\
\hline $\mathrm{T}_{12}-30 \times 20 \mathrm{~cm}+2 \% \mathrm{DAP}$ & 3.83 & 5.10 & 58.3 & 209.9 & 40.5 & 65 & 49 & 26 \\
\hline $\mathrm{T}_{13}-30 \times 30 \mathrm{~cm}+2 \% \mathrm{DAP}$ & 1.95 & 2.68 & 42.1 & 172.3 & 32.8 & 68 & 57 & 27 \\
\hline $\mathrm{T}_{14}-40 \times 20 \mathrm{~cm}+2 \% \mathrm{DAP}$ & 2.11 & 2.52 & 49.8 & 185.3 & 31.3 & 75 & 60 & 33 \\
\hline $\mathrm{T}_{15}-40 \times 30 \mathrm{~cm}+2 \% \mathrm{DAP}$ & 1.53 & 1.90 & 48.9 & 169.3 & 30.1 & 78 & 65 & 35 \\
\hline SEd & 0.10 & 0.12 & 1.6 & 6.0 & 1.6 & 2 & 2 & 1 \\
\hline $\mathrm{CD}(\mathbf{P}=\mathbf{0 . 0 5})$ & 0.22 & 0.25 & 3.41 & 12.3 & 2.37 & 5 & 4 & 2 \\
\hline
\end{tabular}


Table.4 Influence of crop geometry and foliar nutrition on yield parameters and yield of black gram

\begin{tabular}{|c|c|c|c|c|c|c|c|c|}
\hline Treatments & $\begin{array}{l}\text { No. of } \\
\text { pod } \\
\text { clusters } \\
\text { plant }^{-1}\end{array}$ & $\begin{array}{c}\text { No. of } \\
\text { pods } \\
\text { cluster } \\
1\end{array}$ & $\begin{array}{c}\text { Length } \\
\text { of pod } \\
(\mathrm{cm})\end{array}$ & $\begin{array}{l}\text { No. of } \\
\text { Seeds } \\
\text { pod }^{-1}\end{array}$ & $\begin{array}{c}100 \\
\text { seed } \\
\text { weight } \\
(\mathrm{g})\end{array}$ & $\begin{array}{l}\text { Grain } \\
\text { Yield } \\
\text { (kg ha- } \\
{ }^{1} \text { ) }\end{array}$ & $\begin{array}{l}\text { Bhusa } \\
\text { Yield } \\
\text { (kg ha- } \\
{ }^{1} \text { ) }\end{array}$ & $\begin{array}{l}\text { Haulm } \\
\text { Yield } \\
\left(\mathrm{kg} \mathrm{ha}^{-1}\right)\end{array}$ \\
\hline $\mathrm{T}_{1}-30 \times 10 \mathrm{~cm}$ & 7.67 & 3.8 & 4.7 & 5.6 & 4.3 & 817 & 759 & 3438 \\
\hline$T_{2}-30 \times 20 \mathrm{~cm}$ & 13.37 & 4.2 & 4.8 & 5.8 & 4.4 & 814 & 726 & 3166 \\
\hline $\mathrm{T}_{3}-30 \times 30 \mathrm{~cm}$ & 14.64 & 4.4 & 5.0 & 6.0 & 4.5 & 644 & 555 & 2452 \\
\hline$T_{4}-40 \times 20 \mathrm{~cm}$ & 14.32 & 4.3 & 5.5 & 6.0 & 4.5 & 690 & 626 & 2727 \\
\hline $\mathrm{T}_{5}-40 \times 30 \mathrm{~cm}$ & 17.57 & 4.6 & 5.8 & 6.1 & 4.6 & 616 & 542 & 2444 \\
\hline $\mathrm{T}_{6}-30 \times 10 \mathrm{~cm}+1.125 \%$ Pulse Wonder & 7.94 & 4.1 & 5.1 & 6.0 & 4.5 & 990 & 859 & 4048 \\
\hline $\mathrm{T}_{7}-30 \times 20 \mathrm{~cm}+1.125 \%$ Pulse Wonder & 13.95 & 4.5 & 5.3 & 6.2 & 4.6 & 985 & 850 & 3975 \\
\hline $\mathrm{T}_{8}-30 \times 30 \mathrm{~cm}+1.125 \%$ Pulse Wonder & 14.84 & 5.0 & 5.4 & 6.5 & 4.7 & 820 & 751 & 3169 \\
\hline $\mathrm{T}_{9}-40 \times 20 \mathrm{~cm}+1.125 \%$ Pulse Wonder & 16.01 & 4.7 & 6.0 & 6.4 & 4.8 & 880 & 818 & 3703 \\
\hline $\mathrm{T}_{10}-40 \times 30 \mathrm{~cm}+1.125 \%$ Pulse Wonder & 18.52 & 5.2 & 6.2 & 6.6 & 4.8 & 795 & 709 & 3093 \\
\hline $\mathrm{T}_{11}-30 \times 10 \mathrm{~cm}+2 \% \mathrm{DAP}$ & 7.81 & 4.0 & 4.9 & 6.0 & 4.4 & 938 & 829 & 3751 \\
\hline $\mathrm{T}_{12}-30 \times 20 \mathrm{~cm}+2 \% \mathrm{DAP}$ & 13.66 & 4.4 & 5.1 & 6.2 & 4.4 & 932 & 825 & 3683 \\
\hline $\mathrm{T}_{13}-30 \times 30 \mathrm{~cm}+2 \% \mathrm{DAP}$ & 14.76 & 4.7 & 5.2 & 6.4 & 4.6 & 740 & 650 & 2934 \\
\hline $\mathrm{T}_{14}-40 \times 20 \mathrm{~cm}+2 \% \mathrm{DAP}$ & 15.11 & 4.5 & 5.7 & 6.3 & 4.6 & 800 & 694 & 3212 \\
\hline $\mathrm{T}_{15}-40 \times 30 \mathrm{~cm}+2 \% \mathrm{DAP}$ & 18.16 & 4.9 & 6.0 & 6.5 & 4.7 & 723 & 658 & 2972 \\
\hline SEd & 0.007 & 0.16 & 0.17 & 0.06 & 0.16 & 27 & 24 & 106 \\
\hline $\mathrm{CD}(\mathrm{P}=\mathbf{0 . 0 5})$ & 0.016 & 0.32 & 0.36 & 0.11 & NS & 54 & 49 & 218 \\
\hline
\end{tabular}

Table.5 Influence of crop geometry and foliar nutrition on Crop uptak and Post-harvest nutrients of black gram

\begin{tabular}{|c|c|c|c|c|c|c|}
\hline \multirow[t]{2}{*}{ Treatments } & \multicolumn{3}{|c|}{ Nutrient uptake $\left(\mathrm{kg} \mathrm{ha}^{-1}\right)$} & \multicolumn{3}{|c|}{ Available soil nutrients $\left(\mathrm{kg} \mathrm{ha}^{-1}\right)$} \\
\hline & $\mathbf{N}$ & $\mathbf{P}$ & $\mathbf{K}$ & $\mathbf{N}$ & $\mathbf{P}_{2} \mathbf{O}_{5}$ & $\mathbf{K}_{2} \mathbf{O}$ \\
\hline $\mathrm{T}_{1}-30 \times 10 \mathrm{~cm}$ & 63.1 & 10.7 & 54.0 & 272.0 & 13.3 & 267.3 \\
\hline $\mathrm{T}_{2}-30 \times 20 \mathrm{~cm}$ & 58.4 & 10.0 & 50.1 & 275.1 & 13.5 & 273.2 \\
\hline $\mathrm{T}_{3}-30 \times 30 \mathrm{~cm}$ & 41.7 & 7.3 & 36.2 & 278.6 & 13.7 & 279.7 \\
\hline $\mathrm{T}_{4}-40 \times 20 \mathrm{~cm}$ & 47.4 & 8.3 & 41.3 & 282.4 & 14.1 & 286.1 \\
\hline $\mathrm{T}_{5}-40 \times 30 \mathrm{~cm}$ & 43.4 & 7.6 & 37.7 & 285.9 & 14.3 & 292.1 \\
\hline $\mathrm{T}_{6}-30 \times 10 \mathrm{~cm}+1.125 \%$ Pulse Wonder & 75.8 & 12.9 & 64.8 & 288.5 & 14.7 & 271.4 \\
\hline $\mathrm{T}_{7}-30 \times 20 \mathrm{~cm}+1.125 \%$ Pulse Wonder & 72.1 & 12.4 & 61.8 & 293.1 & 15.3 & 277.1 \\
\hline $\mathrm{T}_{8}-30 \times 30 \mathrm{~cm}+1.125 \%$ Pulse Wonder & 58.4 & 10.2 & 50.7 & 298.4 & 15.8 & 283.3 \\
\hline $\mathrm{T}_{9}-40 \times 20 \mathrm{~cm}+1.125 \%$ Pulse Wonder & 66.1 & 11.6 & 57.6 & 304.1 & 16.2 & 289.3 \\
\hline $\mathrm{T}_{10}-40 \times 30 \mathrm{~cm}+1.125 \%$ Pulse Wonder & 56.3 & 9.9 & 49.0 & 309.2 & 16.8 & 296.2 \\
\hline $\mathrm{T}_{11}-30 \times 10 \mathrm{~cm}+2 \% \mathrm{DAP}$ & 70.1 & 11.9 & 59.9 & 279.2 & 14.1 & 269.4 \\
\hline $\mathrm{T}_{12}-30 \times 20 \mathrm{~cm}+2 \% \mathrm{DAP}$ & 68.3 & 11.7 & 58.5 & 283.3 & 14.5 & 275.6 \\
\hline $\mathrm{T}_{13}-30 \times 30 \mathrm{~cm}+2 \% \mathrm{DAP}$ & 53.4 & 9.3 & 46.3 & 288.1 & 14.8 & 281.1 \\
\hline $\mathrm{T}_{14}-40 \times 20 \mathrm{~cm}+2 \% \mathrm{DAP}$ & 57.0 & 10.0 & 49.7 & 292.7 & 15.1 & 287.8 \\
\hline $\mathrm{T}_{15}-40 \times 30 \mathrm{~cm}+2 \% \mathrm{DAP}$ & 53.1 & 9.3 & 46.2 & 297.6 & 15.4 & 293.5 \\
\hline SEd & 1.81 & 0.32 & 1.57 & 44 & 0.5 & 8.7 \\
\hline $\mathrm{CD}(\mathrm{P}=0.05)$ & 3.72 & 0.67 & 3.22 & NS & 1 & 18 \\
\hline
\end{tabular}


Table.6 Influence of crop geometry and foliar nutrition on Crop uptak and Post-harvest nutrients of black gram

\begin{tabular}{|c|c|c|c|c|}
\hline Treatments & $\begin{array}{c}\text { Cost of } \\
\text { cultivation } \\
\left(₹ \text { ha }^{-1}\right)\end{array}$ & $\begin{array}{c}\text { Gross } \\
\text { returns } \\
\left(₹ \text { ha }^{-1}\right)\end{array}$ & $\begin{array}{c}\text { Net } \\
\text { returns (₹ } \\
\left.\text { ha }^{-1}\right)\end{array}$ & $\begin{array}{c}\text { B:C } \\
\text { Ratio }\end{array}$ \\
\hline $\mathrm{T}_{1}-30 \times 10 \mathrm{~cm}$ & 18216 & 38808 & 20592 & 2.13 \\
\hline $\mathrm{T}_{2}-30 \times 20 \mathrm{~cm}$ & 17816 & 38665 & 20849 & 2.17 \\
\hline $\mathrm{T}_{3}-30 \times 30 \mathrm{~cm}$ & 17416 & 30590 & 13174 & 1.76 \\
\hline $\mathrm{T}_{4}-40 \times 20 \mathrm{~cm}$ & 17496 & 32775 & 15279 & 1.87 \\
\hline $\mathrm{T}_{5}-40 \times 30 \mathrm{~cm}$ & 17216 & 29260 & 12044 & 1.70 \\
\hline $\mathrm{T}_{6}-30 \times 10 \mathrm{~cm}+1.125 \%$ Pulse Wonder & 19896 & 47025 & 27129 & 2.36 \\
\hline $\mathrm{T}_{7}-30 \times 20 \mathrm{~cm}+1.125 \%$ Pulse Wonder & 19496 & 46788 & 27292 & 2.40 \\
\hline $\mathrm{T}_{8}-30 \times 30 \mathrm{~cm}+1.125 \%$ Pulse Wonder & 19096 & 38950 & 19854 & 2.04 \\
\hline $\mathrm{T}_{9}-40 \times 20 \mathrm{~cm}+1.125 \%$ Pulse Wonder & 19176 & 41800 & 22624 & 2.18 \\
\hline $\mathrm{T}_{10}-40 \times 30 \mathrm{~cm}+1.125 \%$ Pulse Wonder & 18896 & 37763 & 18867 & 2.00 \\
\hline $\mathrm{T}_{11}-30 \times 10 \mathrm{~cm}+2 \% \mathrm{DAP}$ & 18898 & 44555 & 25657 & 2.36 \\
\hline $\mathrm{T}_{12}-30 \times 20 \mathrm{~cm}+2 \% \mathrm{DAP}$ & 18498 & 44270 & 25772 & 2.39 \\
\hline $\mathrm{T}_{13}-30 \times 30 \mathrm{~cm}+2 \% \mathrm{DAP}$ & 18098 & 35150 & 17052 & 1.94 \\
\hline $\mathrm{T}_{14}-40 \times 20 \mathrm{~cm}+2 \% \mathrm{DAP}$ & 18178 & 38000 & 19822 & 2.09 \\
\hline $\mathrm{T}_{15}-40 \times 30 \mathrm{~cm}+2 \% \mathrm{DAP}$ & 17898 & 34343 & 16445 & 1.92 \\
\hline SEd & - & - & 674 & 0.07 \\
\hline $\mathrm{CD}(\mathrm{P}=0.05)$ & - & - & 1380 & 0.14 \\
\hline
\end{tabular}

In the present study, no striking influence of spacing could be noticed on 100 grain weight as documented in early studies by Luikham $e t$ al., (2009) in broad bean and Shamsi (2009) in chickpea. Foliar spray of $1.125 \%$ TNAU Pulse Wonder recorded the highest yield attributing characters viz., number of pod clusters plant $^{-1}$, number of pods cluster $^{-1}$, number of seeds pod $^{-1}$ and pod length than other foliar spray treatments. The increase in yield attributes might be due to supplementation of nutrients at the critical stage without physiological stress. Foliar application of major and minor nutrients coupled with growth regulators enhanced the number of floral buds, by preventing the floral shedding though maintenance of optimum bio-physiological conditions in plants. Kalpana and Krishnarajan (2003) also reported higher number of pods plant $^{-1}$, number of filled seeds pod $^{-1}$, seed filling percentage and test weight in soybean as a consequence of foliar application of macro and micronutrients along with growth regulators.

\section{Influence of crop geometry and foliar nutrition on yield of blackgram}

Grain yield was significantly higher with closer spacing of $30 \times 10 \mathrm{~cm}$ and $30 \times 20 \mathrm{~cm}$ (Table.4.). This could be due to enhanced vegetative growth and lesser yield attributes owing to severe competition between plants. This is in agreement with the findings of Subramani et al., (2002). Plants under optimum spacing would have effectively utilized the growth resources, particularly solar radiation as compared to plants under narrow spacing. This result is corroborating with the findings of Chaniyara et al., (2002) and Singh et al., (2009) in lentil.

Crop geometry at $30 \times 20 \mathrm{~cm}$ registered higher bhusa and haulm yield as compared to wider spacing, which might be due to more population unit area ${ }^{-1}$. This would have contributed to more biomass and hence higher haulm yield. Such results were also documented earlier by Sathyamoorthi et al., (2008). 
In case of foliar spray, $1.125 \%$ TNAU Pulse Wonder recorded significantly higher grain, bhusa and haulm yield of blackgram as compared to other treatments. The impact of foliar nutrients (macro and micro) to meet the nutrient demand of crop at the critical stage on-site, where they are needed without stress, would have resulted in better growth and development of the crop and ultimately yield attributing characters and yield. The balanced growth habit, which induced more flower and fruiting body production with timely supply of nutrients through foliar spray, might have reduced shedding of flowers and fruits, which led to a positive source-sink gradient of photosynthates translocation due to growth regulators. These favourable effects might have attributed for higher yield of blackgram with foliar spray of nutrients. This findings are in line with the results of Manivannan et al., (2003) who had recorded higher grain yield of blackgram by foliar application of microsol (NPK + micronutrients).

\section{Influence of crop geometry and foliar nutrition on nutrient uptake by blackgram}

Nutrient uptake is a product of nutrient concentration and dry matter accumulation. Crop geometry of $30 \times 10 \mathrm{~cm}$ enhanced the uptake of $\mathrm{N}, \mathrm{P}$ and $\mathrm{K}$ compared to other spacings (Table 5). Better nutrient uptake improved the vegetative growth as indicated by higher dry matter accumulation, resulting in greater DMP at the plant spacing of $30 \times$ $10 \mathrm{~cm}$ than other spacings. This might due to increase in root length that increases the root activity, under higher populations, which enables the increased absorption of nutrients from soil. Large numbers of plants compete for available nutrients unit area ${ }^{-1}$ resulting in higher uptake as documented by Sathyamoorthi et al., (2007) in greengram.

Foliar spray of TNAU Pulse Wonder at $1.125 \%$ concentration enhanced the $\mathrm{N}, \mathrm{P}$ and
K uptake (Fig. 16) which might be attributed to two times of foliar nutritions containing major nutrients providing a balanced nutrient supply. Increased NPK uptake due to foliar application was also observed in greengram by Manu and Wahab (2002).

Influence of crop geometry and foliar nutrition on soil available nutrients after harvest of blackgram

Generally growing of legumes help in nodulation and ultimately enriches soil available N status (Das and Mathur, 1990). Higher post-harvest $\mathrm{N}$ status was observed after the harvest of blackgram. This could be primarily due to increased root nodules and microbial activity in blackgram which might have influenced the nutrient content in the soil. This is in accordance with the results of Manivannan et al., (2002) in blackgram.

Soil available N, P and $\mathrm{K}$ were markedly influenced by different crop geometry and foliar nutrition in blackgram. Higher available $\mathrm{N}, \mathrm{P}$ and $\mathrm{K}$ were recorded at wider spacing of $40 \times 30 \mathrm{~cm}$ ((Table.5.). Depletion of available soil nutrients was noticed at crop geometry of $30 \times 10 \mathrm{~cm}$ spacing. Better growth with higher nutrient availability to crops resulted in increased nutrient uptake of $\mathrm{N}, \mathrm{P}$ and $\mathrm{K}$, thus depleting the soil available nutrients. Under wider spacing, comparatively lesser competition and loss of nutrients from the soil resulted in increased available nutrients. On contrary, Suganthi (2011) reported higher available nutrients with closer spacing in greengram.

Among the various foliar nutrition applied, $1.125 \%$ TNAU Pulse Wonder foliar spray left higher N, P and $\mathrm{K}$ in the soil after harvest of the crop. Higher residual nutrient effect may be attributed to the supply of nutrients through foliar sprays, which reduced the NPK uptake from the soil and thus maintaining 
higher N, P and K status of the soil after the harvest. Further, a vigorous crop growth improves the rooting pattern and higher nodulation and these might have enhanced the atmospheric $\mathrm{N}$ fixation supplementing to the higher value of post-harvest available soil nutrient status.

\section{Influence of crop geometry and foliar nutrition on economics of blackgram cultivation}

Economic efficiency and viability of crop cultivation are mainly the outcome of crop, yield with lesser management cost. Higher crop productivity with less cost of cultivation resulted in better economic parameters like higher net return and $\mathrm{B}$ : $\mathrm{C}$ ratio ((Table.6).

The highest cost of cultivation was observed under crop geometry at $30 \times 10 \mathrm{~cm}$ spacing with foliar nutrition of TNAU Pulse Wonder at $1.125 \%$ (₹ $19,896 \mathrm{ha}^{-1}$ ). Higher grain productivity with $30 \times 10 \mathrm{~cm}$ and $30 \times 20 \mathrm{~cm}$ increased the gross returns ( $₹ 47,025$ and 46,778 ha $^{-1}$ respectively).

The maximum net returns ( $₹ 27,292 \mathrm{ha}^{-1}$ ) and B: C ratio (2.40) were observed under optimum crop geometry of $30 \times 20 \mathrm{~cm}$ with foliar nutrition of TNAU Pulse Wonder at $1.125 \%$. This might be due to the fact that blackgram utilized the resources available below and above the ground very effectively thereby exhibited better growth and yield parameters. The result is supported by the findings of Singh et al., (2009) in blackgram.

Based on the experimental results, it could be concluded that raising of blackgram variety VBN $(\mathrm{Bg}) 5$ with crop geometry at $30 \times 20$ $\mathrm{cm}$ spacing with foliar spray of TNAU Pulse Wonder at $1.125 \%\left(5.625 \mathrm{~kg} \mathrm{ha}^{-1}\right)$ twice at flowering and 15 days later proved to be a better option for getting higher productivity and economics under irrigated conditions.

\section{References}

Anonymous, 2014, http://www.iipr.res.in/pdf/2.2_270615.p df

Anonymous, 2015 http://www.iipr.res.in/pdf/3.15_270615. pdf

Bhairappanavar, S.T., H.M. Jayadeva, T.H. Gowda and S. Shivanna. 2005. Effect of nutrients and spacing on the yield of urdbean under late sown condition. Legume Res., 28 (1): 46-48.

Chaniyara, N.J., R.M. Solanki and V.B. Bhalu. 2002. Effect of inter and intra row spacings on yield of mustard. Agric. Sci. Digest., 22 (1): 48-50.

Cheghakhor, A.Y., M. Meskararbashee, R. Mamaghan and M. Nabipour. 2009. Effect of row spacing and plant density on radiation use efficiency and extinction light coefficient on canopy of chickpea cultivars. Res. Crops, 10 (3): 545-554.

Das, S.K. and B.P. Mathur. 1990. Relative performance of different kharif legumes as pure and intercrops in maize and their residual effect on wheat. Indian J. Agron., 25 (4): 743-745.

Goyal, S., H.D. Verma and D.D. Nawange. 2010. Studies on growth and yield of kabuli Chickpea (Cicer arietinum L.) genotypes under different plant density and fertility levels. Legume Res., 33 (3): 221-223.

Ihsanullah, F.H., Taj, H. Akbar, A. Basir and N. Ullah. 2002. Effect of row spacing on agronomic traits and yield of mungbean (Vigna radiata (L.) Wilczek). Asian J. Plant Sci., 1 (4): 328-329.

ISD (Institute for Sustainable Development). 2009. Piloting a system of crop intensification. In: http://sri.ciifad.cornell.edu/aboutsri/othercr ops/otherSCI/EthSCI_ISD09.pdf.

Jackson, M.L. 1973. Soil chemical analysis. 
Prentice hall of India Pvt. Ltd., New Delhi.

Kalpana, R. and J. Krishnarajan. 2003. Effect of combined application of nutrients and hormones on soybean yield. Legume Res., 26 (2): 151-152.

Kathirvelan, P. and P. Kalaiselvan. 2006. Growth characters, physiological parameters, yield attributes and yield as influenced by the confectionery groundnut varieties and plant population. Res. J. Agric. Bio. Sci., 2 (6): 287-291

Kumar, J. and D. Badiyala. 2005. Influence of seed rate, spacings, date of sowing and storage containers on storability of soybean (Glycine max (L.) Merr.). Legume Res., 28 (4): 309-310.

Luikham, E., M. Phulchand and P.S. M. Anal. 2009. Response of broad bean (Vicia faba L.) to row spacing and phosphorus under late sown rainfed condition of Manipur. Agric. Sci. Digest., 29 (1): 5456.

Manivannan, V., K. Thanunathan, V. Imayavaramban and N. Ramanathan. 2002. Effect of foliar application of NPK and chelated micronutrients on rice-fallow urdbean. Legume Res., 25 (4): $270-272$.

Manivannan, V., K. Thanunathan, V. Imayavaramban and N. Ramanathan. 2003. Growth and growth analysis of rice fallow blackgram as influenced by foliar application of nutrients with and without Rhizobium seed inoculation. Legume Res., 26 (4): 296-299.

Manu, M. and K. Wahab. 2002. Nutrient uptake and nodulation as influenced by irrigation and DAP spray in rice fallow blackgram cv. ADT 5. Plant Archives, 2: 55-57.

Muhammad Amjad, Muhammad Akbar Anjum and Ahmed Ali. 2001. Impact of phosphorus and planting geometry on growth, yield and quality of green pods in okra. Inter J. Agric. Bio., 3 (4): 341344.

Nadeem, M.A., Asghar Ali, Rashid Sohail and Mudassar Maqbool. 2004. Effect of different planting pattern on growth, yield and quality of grain legumes. Pak. J. Life Soc. Sci., 2 (2): 132-135.

Olsen, S.R., C.V. Cole, F.S. Watanabe and L.A. Dean. 1954. Estimation of available phosphorus in soil by extraction with sodium bicarbonate. USDA. Cric., No.939, Washington. $1: 19$.

Santosh Kumar Meena. 2004. Studies on the effect of seed pelleting with nutrients and foliar spray of nutrients and plant growth regulator on growth and yield of irrigated blackgram (Vigna mungo (L.) Hepper). M.Sc. (Ag.) Thesis, Tamil Nadu Agric., Univ., Coimbatore.

Sarkar, A.R., M. H. Kabir, M. Begum and A. Salam. 2004. Yield performance of mungbean as affected by planting date, variety and plant density. J. Agron., 3 (1): 18-24.

Sathyamoorthi, K., M.M. Amanullah, K. Vaiyapuri and E. Somasundaram. 2008. Physiological parameters and yield of greengram (Vigna radiata (L.) Wilczek) as influenced by increased plant density and fertilizer levels. Indian J. Crop. Sci., 3 (1): 115-122.

Sathyamoorthi, K., M.M. Amanullah, K. Vaiyapuri and E. Somasundaram. 2007. Influence of increased plant density and fertilizer application on the nutrient uptake and yield of greengram (Vigna radiata (L.) Wilczek). Res. J. Agric. Bio. Sci., 3 (6): 886-895

Shamsi, K. 2009. Effect of sowing date and row spacing on yield and yield components of chickpea under rainfed Conditions in Iran. J. Appl. Bio. Sci., 17: 941-947.

Siddaraju, R., S. Narayanaswamy, Ramegowda and S. R. Prasad. 2010. 
Studies on growth, seed yield and yield attributes as influenced by varieties and row spacing in cluster bean (Cyamopsis tetragonoloba L.). Mysore J. Agric. Sci., 44 (1): 16-21.

Siddaraju, R., S. Narayanaswamy, Ramegowda and S. R. Prasad. 2010. Studies on growth, seed yield and yield attributes as influenced by varieties and row spacing in cluster bean (Cyamopsis tetragonoloba L.). Mysore J. Agric. Sci., 44 (1): 16-21.

Singh, P., S. Elamathi and P. Ananthi. 2009. Effect of row spacing and dates of sowing on growth and yield of lentil (Lens culinaris) under north eastern region of Uttar Pradesh. Legume Res., 32 (4): 307- 308 .

Stanford, S. and L. English. 1949. Use of flame photometer in rapid soil tests of $\mathrm{K}$ and Ca. Agron. J., 41: 446-447.

Subbiah, B.V. and G.L. Asija. 1956. A rapid procedure for the estimation of available nitrogen in soils. Curr. Sci., 25: $259-260$.

Subramani, M., A. Solaimalai and A.Velayutham. 2002. Effect of plant population and methods of fertilizer application on yield attributes of irrigated blackgram. Madras Agric. J., 89 (4-6): 305-306.

Suganthi, S. 2011. Study on the effect of planting geometry and foliar application of nutrients on greengram. M.Sc. (Ag.) Thesis, Tamil Nadu Agric. Univ., Coimbatore.

Uphoff, N., Marguerite, J. Devi, D. Behera, A.K. Verma and B.J. Pandian. 2011. National Colloquium on System of Crop Intensification (SCI). In: http://sri.ciifad.cornell. edu/aboutsri/othercrops/index.html.

Vijayakumar, M., S. Ramesh, B. Chandrasekaran and T.M. Thiyagarajan. 2006. Effect of System of rice intensification (SRI) practices on yield attributes, yield and water productivity of rice (Oryza sativa L.). Res. J. Agric. Biol. Sci., 2 (6): 236-242.

Walkley, A. and C.A. Black. 1934. An estimation of wet oxidation method for determining soil organic matter and proposed modification of chromic acid titration method. Soil Sci., 31: 29-34.

\section{How to cite this article:}

Rajeshkumar, S., S. Nalliah Durairaj and Kannan, V. 2017. Effect of Crop Geometry and Foliar Nutrition on Growth and Yield of Irrigated Blackgram (Vigna mungo L.). Int.J.Curr.Microbiol.App.Sci. 6(11): 4084-4094. doi: https://doi.org/10.20546/ijcmas.2017.611.478 